\title{
Hermite-Hadamard type inequalities, convex stochastic processes and Katugampola fractional integral
}

\author{
Jorge E. Hernández H. ${ }^{a *}$, JuAn Francisco Gómez ${ }^{b}$ \\ ${ }^{a}$ Universidad Centroccidental Lisandro Alvarado, Decanato de Ciencias Económicas \\ y Empresariales, Barquisimeto, Venezuela. \\ ${ }^{b}$ Universidad Centroccidental Lisandro Alvarado, Dirección del Centro de \\ Investigaciones del DCEE, Barquisimeto, Venezuela.
}

\begin{abstract}
In this work we present some Hermite-Hadamard type ine-qualities for convex Stochastic Processes using the Katugampola fractional integral, and from these results specific cases are deduced for the Riemann-Liouville fractional integral and Riemann integral. Also, a refinement of the aforementioned inequality is presented.

Keywords: Hermite-Hadamard inequality, Stochastic Processes, Fractional Integrals.

MSC2010: 60E15, 26B25, 26A33.
\end{abstract}

\section{Desigualdades de tipo Hermite-Hadamard, procesos estocásticos convexos y la integral fraccionaria de Katugampola}

Resumen. En este trabajo se presentan algunas desigualdades de tipo Hermite-Hadamard para procesos estocásticos convexos usando la integral fraccional de Katugampola, y de estos resultados se deducen casos específicos para la integral fraccionaria de Riemann-Liouville y la integral de Riemann. Palabras clave: Desigualdad de Hermite-Hadamard, Procesos Estocásticos, Integral fraccionaria de Katugampola.

\footnotetext{
*E-mail: jorgehernandez@ucla.edu.ve

Received: 11 July 2018, Accepted: 13 November 2018.

To cite this article: J.E. Hernández H., J.F. Gómez, Hermite-Hadamard type inequalities, convex stochastic processes and Katugampola fractional integral, Rev. Integr. temas mat. 36 (2018), No. 2, 133-149. doi: $10.18273 /$ revint.v36n2-2018005
} 


\section{Introduction}

The study of convex functions has been of interest for mathematical analysis based on the properties that are deduced from this concept. Due to generalization requirements of the convexity concept in order to obtain new applications, in the last years great efforts have been made in the study and investigation of this topic.

A function $f: I \rightarrow \mathbb{R}$ is said to be convex if for all $x, y \in I$ and $t \in[0,1]$ the inequality

$$
f(t x+(1-t) y) \leq t f(x)+(1-t) f(y)
$$

holds.

Numerous works of investigation have been realized extending results on inequalities for convex functions towards others much more generalized, using new concepts such as $E$-convexity [35], quasi-convexity [28], $s$-convexity [3], logarithmically convexity [2], and others.

A compendium about the history of the Hermite-Hadamard inequality can be found in the work of D.S. Mitrinovic and I.B. Lackovic [22]. The formulation of this result is as follows:

(Hermite-Hadamard Inequality). Let $f: I \rightarrow \mathbb{R}$ be a convex function, and $a, b \in I$ with $a<b$, then

$$
f\left(\frac{a+b}{2}\right) \leq \frac{1}{b-a} \int_{a}^{b} f(x) d x \leq \frac{f(a)+f(b)}{2} .
$$

The inequality of Hermite-Hadamard has become a very useful tool in the Theory of Probability and Optimization (See [18]).

The study on convex stochastic processes began in 1974 when B. Nagy, in [23], applied a characterization of measurable stochastic processes to solving a generalization of the (additive) Cauchy functional equation. In 1980 Nikodem [24] considered convex stochastic processes. In 1992 and 1995 Skowronski [31], [32] obtained some further results on convex stochastic processes which generalize some known properties.

In the year 2014, E. Set et. al. in [27] investigated Hermite-Hadamard type inequalities for stochastic processes in the second sense (For other results related to stochastic processes see [4], [9], [20], [29], [30], where further references are given).

Also, Fractional calculus [10], [21] was introduced at the end of the nineteenth century by Liouville and Riemann, the subject of which has become a rapidly growing area and has found applications in diverse fields ranging from physical sciences and engineering to biological sciences and economics.

In 2011, U. Katugampola presented a new fractional integral operator in [12], which generalizes the Riemann-Liouville and the Hadamard integrals into a single form, and various researchers have made use of this result in the field of convexity, generalized convexity and others ([5], [7], [8], [33]).

Recently, several Hermite-Hadamard type inequalities [19], [34] associated with fractional integrals have been investigated. Here, it is established some generalized HermiteHadamard type integral inequalities for stochastic processes using Katugampola fractional integral operator, which generalize, in a single form those found using RiemannLiouville fractional integral and Hadamard fractional integral. Also, it is proposed a 
refinement of the inequality object of study using the aforementioned fractional integral. Application areas of the results found are optimization, especially in optimal designs, and also useful for numerical approximations when there exist probabilistic quantities [29].

\section{Preliminaries}

\subsection{About calculus of stochastic processes.}

The following notions corresponds to ordinary and convex Stochastic Process (References about can be found in [16], [17], [20], [31], [32].

Definition 2.1. Let $(\Omega, \mathcal{A}, P)$ be an arbitrary probability space. A function $X: \Omega \rightarrow \mathbb{R}$ is called a random variable if it is $\mathcal{A}$-measurable. Let $I \subset \mathbb{R}$ be an interval indicating time. A function $X: I \times \Omega \rightarrow \mathbb{R}$ is called a stochastic process if for every $t \in I$ the function $X(t, \cdot)$ is a random variable.

1. If $X(t, w)$ takes values in $\mathbb{R}^{n}$ it is called vector-valued stochastic process.

2. If the time $I$ can be a discrete subset of $\mathbb{R}$, then $X(t, w)$ is called a discrete time stochastic process.

3. If the time $I$ is an interval, $\mathbb{R}^{+}$or $\mathbb{R}$, it is called a stochastic process with continuous time.

Definition 2.2. Let $(\Omega, A, P)$ be a probability space and $I \subset \mathbb{R}$ be an interval. A stochastic process $X: I \times \Omega \rightarrow \mathbb{R}$ is called:

1. Increasing (decreasing) if for all $u, v \in I$ such that $u<v$,

$$
X(u, \cdot) \leq X(v, \cdot), \quad(X(u, \cdot) \geq X(v, \cdot)), \quad(\text { a.e. })
$$

2. Monotonic, if it's increasing or decreasing;

3. Continuous in probability in the interval $I$, if for all $t_{0} \in I$ the following limit holds:

$$
P-\lim _{t \rightarrow t_{0}} X(t, \cdot)=X\left(t_{0}, \cdot\right),
$$

where $P$ - lim denotes the limit in probability;

4. Mean square continuous in the interval $I$, if the limit for all $t_{0} \in I$

$$
\lim _{t \rightarrow t_{0}} E\left[X(t, \cdot)-X\left(t_{0}, \cdot\right)\right]^{2}=0,
$$

where $E[X(t, \cdot)]$ denotes the expectation value of the random variable $X(t, \cdot)$;

5. Mean square differentiable in $I$, if there exist a stochastic process $X^{\prime}(t, \cdot)$ (the derivative of $X$ ) such that for all $t_{0} \in I$ we have

$$
\lim _{t \rightarrow t_{0}} E\left[\frac{X(t, \cdot)-X\left(t_{0}, \cdot\right)}{t-t_{0}}-X^{\prime}\left(t_{0}, \cdot\right)\right]^{2}=0
$$

Vol. 36, $\left.\mathrm{N}^{\circ} 2,2018\right]$ 
Definition 2.3. Let $(\Omega, A, P)$ be a probability space, $I \subset \mathbb{R}$ be an interval with $E[X(t)]^{2}<\infty$ for all $t \in I$.

Let $[a, b] \subset I, a=t_{0}<t_{1}<\ldots<t_{n}=b$ be a partition of $[a, b]$ and $\theta_{k} \in\left[t_{k-1}, t_{k}\right]$ for $k=1,2, \ldots, n$.

A random variable $Y: \Omega \rightarrow \mathbb{R}$ is called mean-square integral of the process $X(t, \cdot)$ on $[a, b]$, if the following identity holds:

$$
\lim _{n \rightarrow \infty} E\left[\sum_{k=1}^{n} X\left(\theta_{k}, \cdot\right)\left(t_{k}-t_{k-1}\right)-Y(\cdot)\right]^{2}=0 ;
$$

in such a way, it can be written

$$
\int_{a}^{b} X(t, \cdot) d t=Y(\cdot) \quad(\text { a.e. })
$$

Also, mean square integral operator is increasing, that is,

$$
\int_{a}^{b} X(t, \cdot) d t \leq \int_{a}^{b} Z(t, \cdot) d t \quad(\text { a.e. }),
$$

where $X(t, \cdot) \leq Z(t, \cdot)$ in $[a, b]$.

Throughout this paper, it will be considered mean square continuous stochastic processes. Important theorems as the mean value theorem for mean square derivatives and integrals for stochastic processes have been proved in the work of J.C. Cortés et. al. The reader can find these results in [6, Lemma 3.1, Theorem 3.2].

In 1980 K. Nikodem introduced the following definition [24].

Definition 2.4. Set $(\Omega, \mathcal{A}, P)$ be a probability space and $I \subset \mathbb{R}$ be an interval. The stochastic process $X: I \times \Omega \rightarrow \mathbb{R}$ is said to be a convex stochastic process if

$$
X(\lambda u+(1-\lambda) v, \cdot) \leq \lambda X(u, \cdot)+(1-\lambda) X(v, \cdot)
$$

holds almost everywhere for all $u, v \in I$ and $\lambda \in[0,1]$.

One of the results of interest for the present work is the following.

Theorem 2.5. Every Jensen-convex stochastic process and continuous in probability is convex.

Using Definition 2.4, D. Kotrys presented, in 2012, the Hermite-Hadamard integral inequality version for Stochastic Processes [16].

Theorem 2.6. If $X: I \times \Omega \rightarrow \mathbb{R}$ is convex and mean square continuous in the interval $T \times \Omega$, then for any $u, v \in T$, the inequality

$$
X\left(\frac{u+v}{2}, \cdot\right) \leq \frac{1}{u-v} \int_{u}^{v} X(t, \cdot) d t \leq \frac{X(u, \cdot)+X(v, \cdot)}{2}
$$

holds almost everywhere.

[Revista Integración, temas de matemáticas 


\subsection{About generalized fractional integral operators}

Before establishing the main results, it will be given some necessary notions and mathematical preliminaries of fractional calculus theory which are used further in this paper. For more details, consult [10], [15], [21], [25].

Definition 2.7. Let $f \in L_{1}([a, b])$. The Riemann-Liouville integrals $J_{a+}^{\alpha}$ and $J_{b-}^{\alpha}$ of order $\alpha>0$ with $a \geq 0$ are defined by

$$
J_{a+}^{\alpha} f(x)=\frac{1}{\Gamma(\alpha)} \int_{a}^{x}(x-t)^{\alpha-1} f(t) d t
$$

and

$$
J_{b-}^{\alpha} f(x)=\frac{1}{\Gamma(\alpha)} \int_{x}^{b}(t-x)^{\alpha-1} f(t) d t,
$$

respectively, where $\Gamma(\alpha)$ is the Euler's Gamma function defined by

$$
\Gamma(\alpha)=\int_{0}^{\infty} t^{\alpha-1} e^{-t} d t
$$

Note that $J_{a+}^{0} f(x)=J_{b-}^{0} f(x)=f(x)$.

Using the Riemann-Liouville fractional integral, Sarikaya et all [26] established the Hermite-Hadamard inequalities version.

Theorem 2.8. Let $f:[a, b] \rightarrow \mathbb{R}$ be a positive function with $a<b$ and $f \in L_{1}([a, b])$. If $f$ is a convex function on $[a, b]$, then, with $\alpha>0$,

$$
f\left(\frac{a+b}{2}\right) \leq \frac{\Gamma(\alpha+1)}{(b-a)^{\alpha}}\left(J_{a+}^{\alpha} f(b)+J_{b-}^{\alpha} f(a)\right) \leq \frac{f(a)+f(b)}{2} .
$$

Also, J. Hadamard in 1892 introduced the following fractional integral operator ([11]).

Definition 2.9. Let $\alpha>0$ with $n-1<\alpha<n, n \in \mathbb{N}$, and $a<x<b$. The left and right-side Hadamard fractional integrals of order $\alpha>0$ of a function $f$, are given by

$$
H_{a+}^{\alpha} f(t)=\frac{1}{\Gamma(\alpha)} \int_{a}^{x}\left(\ln \frac{x}{t}\right)^{\alpha-1} \frac{f(t)}{t} d t
$$

and

$$
H_{b-}^{\alpha} f(t)=\frac{1}{\Gamma(\alpha)} \int_{x}^{b}\left(\ln \frac{x}{t}\right)^{\alpha-1} \frac{f(t)}{t} d t
$$

respectively.

As it was mentioned in the introductory section, Katugampola introduced a new fractional integral that generalizes the Riemann-Liouville and Hadamard fractional integrals into a single form (see [12], [13], [14]).

Vol. 36, $\left.\mathrm{N}^{\circ} 2,2018\right]$ 
In the following will denote the space $X_{c}^{p}(a, b),(c \in \mathbb{R}, 1 \leq p \leq \infty)$ of those complex valued Lebesgue measurable functions $f$ on $[a, b]$ for which $\|f\|_{X_{c}^{p}}<\infty$, where

$$
\|f\|_{X_{c}^{p}}=\left(\int_{a}^{b}\left|t^{c} f(t)\right|^{p} \frac{d t}{t}\right)^{1 / p} .
$$

Katugampola in [13] established the following definition and property.

Definition 2.10. Let $[a, b] \subset \mathbb{R}$ be a finite interval. The left and right sides of Katugampola fractional integral of order $\alpha>0$ of $f \in X_{c}^{p}(a, b)$ are defined by

$$
{ }^{\rho} I_{a+}^{\alpha} f(x)=\frac{\rho^{1-\alpha}}{\Gamma(\alpha)} \int_{a}^{x} \frac{t^{\rho-1}}{\left(x^{\rho}-t^{\rho}\right)^{1-\alpha}} f(t) d t
$$

and

$$
{ }^{\rho} I_{b^{-}}^{\alpha} f(x)=\frac{\rho^{1-\alpha}}{\Gamma(\alpha)} \int_{x}^{b} \frac{t^{\rho-1}}{\left(t^{\rho}-x^{\rho}\right)^{1-\alpha}} f(t) d t,
$$

respectively, with $a<x<b$ and $\rho>0$, if the integrals exist.

Theorem 2.11. Let $\alpha>0$ and $\rho>0$. Then, for $x>a$,

$$
\lim _{\rho \rightarrow 1}{ }^{\rho} I_{a+}^{\alpha} f(x)=J_{a+}^{\alpha} f(x)
$$

and

$$
\lim _{\rho \rightarrow 0^{+}}{ }^{\rho} I_{a+}^{\alpha} f(x)=H_{a+}^{\alpha} f(x) .
$$

Similar results also hold for the right-sided operators.

The purpose of this paper is to derive some inequalities of type Hermite-Hadamard for convex stochastic processes using the Katugampola fractional integrals.

\section{Main Results}

Theorem 3.1. Let $\alpha>0$ and $\rho>0$. Let $X:\left[a^{\rho}, b^{\rho}\right] \times \Omega \rightarrow \mathbb{R}$ be a positive stochastic process with $0 \leq a<b$ and $X(t, \cdot) \in X_{c}^{p}\left(a^{\rho}, b^{\rho}\right)$. If $X(t, \cdot)$ is convex, the following inequality holds almost everywhere:

$$
\begin{aligned}
X\left(\frac{a^{\rho}+b^{\rho}}{2}, \cdot\right) & \leq \frac{\Gamma(\alpha+1)}{2 \rho^{-\alpha}\left(b^{\rho}-a^{\rho}\right)^{\alpha}}\left({ }^{\rho} I_{b^{\rho}-}^{\alpha} X\left(a^{\rho}, \cdot\right)+{ }^{\rho} I_{a^{\rho}+}^{\alpha} X\left(b^{\rho}, \cdot\right)\right) \\
& \leq \frac{X\left(a^{\rho}, \cdot\right)+X\left(b^{\rho}, \cdot\right)}{2 \rho \alpha} .
\end{aligned}
$$

Proof. Let $t \in[0,1]$, and $u, v \in[a, b]$ defined by

$$
u^{\rho}=t^{\rho} a^{\rho}+\left(1-t^{\rho}\right) b^{\alpha} \text { and } v^{\rho}=\left(1-t^{\rho}\right) a^{\rho}+t^{\rho} b^{\rho} .
$$


Since $X$ is a convex stochastic process,

$$
X\left(\frac{u^{\rho}+v^{\rho}}{2}, \cdot\right) \leq \frac{X\left(u^{\rho}, \cdot\right)+X\left(v^{\alpha}, \cdot\right)}{2} ;
$$

using (3), it can be rewritten as

$$
2 X\left(\frac{a^{\rho}+b^{\rho}}{2}, \cdot\right) \leq X\left(t^{\rho} a^{\rho}+\left(1-t^{\rho}\right) b^{\alpha}, \cdot\right)+X\left(\left(1-t^{\rho}\right) a^{\rho}+t^{\rho} b, \cdot\right) .
$$

Multiplying both sides of (4) by $t^{\alpha \rho-1},(\alpha, \rho>0)$ and integrating over $t \in[0,1]$, it is obtained that

$$
\begin{aligned}
\frac{2}{\alpha \rho} X\left(\frac{a^{\rho}+b^{\rho}}{2}, \cdot\right) \leq \int_{0}^{1} t^{\alpha \rho-1} X\left(t^{\rho} a^{\rho}+(1\right. & \left.\left.-t^{\rho}\right) b^{\alpha}, \cdot\right) d t \\
& +\int_{0}^{1} t^{\alpha \rho-1} X\left(\left(1-t^{\rho}\right) a^{\rho}+t^{\rho} b^{\rho}, \cdot\right) d t .
\end{aligned}
$$

Now, from (3) and the Definition 2.10, it is obtained that

$$
\begin{aligned}
\int_{0}^{1} t^{\alpha \rho-1} X\left(t^{\rho} a^{\rho}+\left(1-t^{\rho}\right) b^{\alpha}, \cdot\right) d t & =\frac{1}{\left(b^{\rho}-a^{\rho}\right)^{\alpha}} \int_{a}^{b} \frac{u^{\rho-1}}{\left(u^{\rho}-b^{\rho}\right)^{1-\alpha}} X\left(u^{\rho}, \cdot\right) d u \\
& =\frac{\Gamma(\alpha)}{\rho^{1-\alpha}\left(b^{\rho}-a^{\rho}\right)^{\alpha}}{ }^{\rho} I_{b-}^{\alpha} X\left(a^{\rho}, \cdot\right)
\end{aligned}
$$

and

$$
\begin{aligned}
\int_{0}^{1} t^{\alpha \rho-1} X\left(\left(1-t^{\rho}\right) a^{\rho}+t^{\rho} b^{\rho}, \cdot\right) d t & =\frac{1}{\left(b^{\rho}-a^{\rho}\right)^{\alpha}} \int_{a}^{b} \frac{v^{\rho-1}}{\left(b^{\rho}-v^{\rho}\right)^{1-\alpha}} X\left(v^{\rho}, \cdot\right) d v \\
& =\frac{\Gamma(\alpha)}{\rho^{1-\alpha}\left(b^{\rho}-a^{\rho}\right)^{\alpha}}{ }^{\rho} I_{a+}^{\alpha} X\left(b^{\rho}, \cdot\right) .
\end{aligned}
$$

Replacing (6) and (7) in (5), it is obtained the left side of the inequality (2)

$$
X\left(\frac{a^{\rho}+b^{\rho}}{2}, \cdot\right) \leq \frac{\Gamma(\alpha+1)}{2 \rho^{-\alpha}\left(b^{\rho}-a^{\rho}\right)^{\alpha}}\left({ }^{\rho} I_{b^{\rho}-}^{\alpha} X\left(a^{\rho}, \cdot\right)+{ }^{\rho} I_{a^{\rho}+}^{\alpha} X\left(b^{\rho}, \cdot\right)\right) .
$$

In order to obtain the right side of the inequality(2), it is used the convex property of the stochastic process $X$ :

$$
\begin{aligned}
& X\left(t^{\rho} a^{\rho}+\left(1-t^{\rho}\right) b^{\alpha}, \cdot\right) \leq t^{\rho} X\left(a^{\rho}, \cdot\right)+\left(1-t^{\rho}\right) X\left(b^{\rho}, \cdot\right), \\
& X\left(\left(1-t^{\rho}\right) a^{\rho}+t^{\rho} b^{\rho}, \cdot\right) \leq\left(1-t^{\rho}\right) X\left(a^{\rho}, \cdot\right)+t^{\rho} X\left(b^{\rho}, \cdot\right) ;
\end{aligned}
$$

adding these inequalities it is obtained

$$
X\left(t^{\rho} a^{\rho}+\left(1-t^{\rho}\right) b^{\alpha}, \cdot\right)+X\left(\left(1-t^{\rho}\right) a^{\rho}+t^{\rho} b^{\rho}, \cdot\right) \leq X\left(a^{\rho}, \cdot\right)+X\left(b^{\rho}, \cdot\right) .
$$

Multiplying both sides of (8) by $t^{\alpha \rho-1},(\alpha, \rho>0)$ and integrating over $t \in[0,1]$, it is attained that

$$
\frac{\Gamma(\alpha)}{2 \rho^{-\alpha}\left(b^{\rho}-a^{\rho}\right)^{\alpha}}\left({ }^{\rho} I_{b^{\rho}-}^{\alpha} X\left(a^{\rho}, \cdot\right)+{ }^{\rho} I_{a^{\rho}+}^{\alpha} X\left(b^{\rho}, \cdot\right)\right) \leq \frac{X\left(a^{\rho}, \cdot\right)+X\left(b^{\rho}, \cdot\right)}{2 \rho \alpha} .
$$

The proof is complete. 
Remark 3.2. Using the Theorem 2.11 we get the Hermite Hadamard inequality version for the Riemann Liouville fractional integral,

$$
X\left(\frac{a+b}{2}, \cdot\right) \leq \frac{\Gamma(\alpha+1)}{2(b-a)^{\alpha}}\left(J_{b-}^{\alpha} X(a, \cdot)+J_{a-}^{\alpha} X(b, \cdot)\right) \leq \frac{X(a, \cdot)+X(b, \cdot)}{2 \alpha},
$$

almost everywhere, making coincidence with the result proved by $\mathrm{H}$. Aghahi and A. Babakhani in [1]. Letting $\alpha=1$ in (9), it is obtained the Hermite Hadamard inequality for the ordinary Riemann integral

$$
X\left(\frac{a+b}{2}, \cdot\right) \leq \frac{1}{(b-a)} \int_{a}^{b} X(t, \cdot) d t \leq \frac{X(a, \cdot)+X(b, \cdot)}{2}, \quad \text { (a.e.) }
$$

making coincidence with the result proved by Kotrys in [16].

Theorem 3.3. Let $X:\left[a^{\rho}, b^{\rho}\right] \times \Omega \rightarrow \mathbb{R}$ be a square mean differentiable stochastic process with $0 \leq a<b$. If $X^{\prime}$ is a square mean differentiable stochastic process, then the following inequality holds almost everywhere:

$$
\begin{aligned}
\mid \frac{X\left(a^{\rho}, \cdot\right)+X\left(b^{\rho}, \cdot\right)}{2}-\frac{\alpha \rho^{\alpha} \Gamma(\alpha+1)}{2\left(b^{\rho}-a^{\rho}\right)^{\alpha}} & \left({ }^{\rho} I_{b^{\rho}-}^{\alpha} X\left(a^{\rho}, \cdot\right)+{ }^{\rho} I_{a^{\rho}+}^{\alpha} X\left(b^{\rho}, \cdot\right)\right) \mid \\
\leq & \frac{\left(b^{\rho}-a^{\rho}\right)^{2}}{2(\alpha+1)(\alpha+2)}\left(\alpha+\frac{1}{2^{\alpha}}\right) \sup _{\xi \in\left[a^{\rho}, b^{\rho}\right]}\left|X^{\prime \prime}(\xi, \cdot)\right| .
\end{aligned}
$$

Proof. From the proof of Theorem 3.1 we get

$$
\begin{aligned}
& \frac{\Gamma(\alpha+1)}{2 \rho^{1-\alpha}\left(b^{\rho}-a^{\rho}\right)^{\alpha}}\left({ }^{\rho} I_{b^{\rho}-}^{\alpha} X\left(a^{\rho}, \cdot\right)+{ }^{\rho} I_{a^{\rho}+}^{\alpha} X\left(b^{\rho}, \cdot\right)\right) \\
& \quad=\int_{0}^{1} t^{\alpha \rho-1} X\left(t^{\rho} a^{\rho}+\left(1-t^{\rho}\right) b^{\alpha}, \cdot\right) d t+\int_{0}^{1} t^{\alpha \rho-1} X\left(\left(1-t^{\rho}\right) a^{\rho}+t^{\rho} b^{\rho}, \cdot\right) d t .
\end{aligned}
$$

Integrating by parts each of the integrals we have

$$
\begin{aligned}
\int_{0}^{1} & t^{\alpha \rho-1} X\left(t^{\rho} a^{\rho}+\left(1-t^{\rho}\right) b^{\alpha}, \cdot\right) d t \\
& =\left.\frac{t^{\alpha \rho} X\left(t^{\rho} a^{\rho}+\left(1-t^{\rho}\right) b^{\alpha}, \cdot\right)}{\alpha \rho}\right|_{0} ^{1}-\frac{\left(a^{\rho}-b^{\rho}\right)}{\alpha} \int_{0}^{1} t^{\alpha \rho+\rho-1} X^{\prime}\left(t^{\rho} a^{\rho}+\left(1-t^{\rho}\right) b^{\alpha}, \cdot\right) d t \\
& =\frac{X\left(a^{\rho}, \cdot\right)}{\alpha \rho}-\frac{\left(a^{\rho}-b^{\rho}\right)}{\alpha} \int_{0}^{1} t^{\rho(\alpha+1)-1} X^{\prime}\left(t^{\rho} a^{\rho}+\left(1-t^{\rho}\right) b^{\alpha}, \cdot\right) d t
\end{aligned}
$$

and

$$
\begin{aligned}
& \int_{0}^{1} t^{\alpha \rho-1} X\left(\left(1-t^{\rho}\right) a^{\rho}+t^{\rho} b^{\rho}, \cdot\right) d t \\
& \quad=\left.\frac{t^{\alpha \rho} X\left(\left(1-t^{\rho}\right) a^{\rho}+t^{\rho} b^{\rho}, \cdot\right)}{\alpha \rho}\right|_{0} ^{1}-\frac{\left(b^{\rho}-a^{\rho}\right)}{\alpha} \int_{0}^{1} t^{\alpha \rho+\rho-1} X^{\prime}\left(\left(1-t^{\rho}\right) a^{\rho}+t^{\rho} b^{\rho}, \cdot\right) d t \\
& \quad=\frac{X\left(b^{\rho}, \cdot\right)}{\alpha \rho}-\frac{\left(b^{\rho}-a^{\rho}\right)}{\alpha} \int_{0}^{1} t^{\rho(\alpha+1)-1} X^{\prime}\left(\left(1-t^{\rho}\right) a^{\rho}+t^{\rho} b^{\rho}, \cdot\right) d t
\end{aligned}
$$

[Revista Integración, temas de matemáticas 
So,

$$
\begin{aligned}
& \frac{\Gamma(\alpha+1)}{2 \rho^{1-\alpha}\left(b^{\rho}-a^{\rho}\right)^{\alpha}}\left({ }^{\rho} I_{b^{\rho}-}^{\alpha} X\left(a^{\rho}, \cdot\right)+{ }^{\rho} I_{a^{\rho}+}^{\alpha} X\left(b^{\rho}, \cdot\right)\right) \\
& \quad=\frac{X\left(a^{\rho}, \cdot\right)+X\left(b^{\rho}, \cdot\right)}{\alpha \rho}-\frac{\left(b^{\rho}-a^{\rho}\right)}{\alpha} \times \\
& \quad\left(\int_{0}^{1} t^{\rho(\alpha+1)-1}\left(X^{\prime}\left(t^{\rho} a^{\rho}+\left(1-t^{\rho}\right) b^{\alpha}, \cdot\right)-X^{\prime}\left(\left(1-t^{\rho}\right) a^{\rho}+t^{\rho} b^{\rho}, \cdot\right)\right) d t\right) .
\end{aligned}
$$

Applying the Mean Value Theorem for $X^{\prime}$ it is obtained

$$
\begin{aligned}
& \frac{\Gamma(\alpha+1)}{2 \rho^{1-\alpha}\left(b^{\rho}-a^{\rho}\right)^{\alpha}}\left({ }^{\rho} I_{b^{\rho}-}^{\alpha} X\left(a^{\rho}, \cdot\right)+{ }^{\rho} I_{a^{\rho}+}^{\alpha} X\left(b^{\rho}, \cdot\right)\right) \\
& =\frac{X\left(a^{\rho}, \cdot\right)+X\left(b^{\rho}, \cdot\right)}{\alpha \rho}-\frac{\left(b^{\rho}-a^{\rho}\right)^{2}}{\alpha}\left(\int_{0}^{1} t^{\rho(\alpha+1)-1}\left(2 t^{\rho}-1\right) X^{\prime \prime}(\xi, \cdot) d t\right)
\end{aligned}
$$

for some $\xi \in\left[a^{\rho}, b^{\rho}\right]$.

Now, it can be written

$$
\begin{aligned}
& \left|\frac{X\left(a^{\rho}, \cdot\right)+X\left(b^{\rho}, \cdot\right)}{\alpha \rho}-\frac{\Gamma(\alpha+1)}{2 \rho^{1-\alpha}\left(b^{\rho}-a^{\rho}\right)^{\alpha}}\left({ }^{\rho} I_{b^{\rho}-}^{\alpha} X\left(a^{\rho}, \cdot\right)+{ }^{\rho} I_{a^{\rho}+}^{\alpha} X\left(b^{\rho}, \cdot\right)\right)\right| \\
& \quad \leq \frac{\left(b^{\rho}-a^{\rho}\right)^{2}\left|X^{\prime \prime}(\xi, \cdot)\right|}{\alpha}\left(\int_{0}^{1} t^{\rho(\alpha+1)-1}\left|2 t^{\rho}-1\right| d t\right) \\
& \quad=\frac{\left(b^{\rho}-a^{\rho}\right)^{2}\left|X^{\prime \prime}(\xi, \cdot)\right|}{\alpha} \times \\
& \quad\left(\int_{0}^{1 / 2^{1 / \rho}} t^{\rho(\alpha+1)-1}\left(1-2 t^{\rho}\right) d t+\int_{1 / 2^{1 / \rho}}^{1} t^{\rho(\alpha+1)-1}\left(2 t^{\rho}-1\right) d t\right) \\
& \quad \leq \frac{\left(b^{\rho}-a^{\rho}\right)^{2}}{\alpha \rho(\alpha+1)(\alpha+2)}\left(\alpha+\frac{1}{2^{\alpha}}\right) \sup _{\xi \in\left[a^{\rho}, b^{\rho}\right]}\left|X^{\prime \prime}(\xi, \cdot)\right| .
\end{aligned}
$$

The proof is complete.

Remark 3.4. Using Theorem 2.11 and taking limit when $\rho \rightarrow 1$ in Theorem 3.3, it is obtained the version for the Riemann-Liouville fractional integral

$$
\begin{aligned}
\mid \frac{X\left(a^{\rho}, \cdot\right)+X\left(b^{\rho}, \cdot\right)}{2}- & \frac{\Gamma(\alpha+1)}{2(b-a)^{\alpha}}\left(J_{b-}^{\alpha} X\left(a^{\rho}, \cdot\right)+J_{a+}^{\alpha} X\left(b^{\rho}, \cdot\right)\right) \mid \\
& \left.\leq \frac{(b-a)^{2}}{2(\alpha+1)(\alpha+2)}\left(\alpha+\frac{1}{2^{\alpha}}\right) \sup _{\xi \in[a, b]}\left|X^{\prime \prime}(\xi, \cdot)\right|, \quad \text { (a.e. }\right)
\end{aligned}
$$

and letting $\alpha=1$ in (12) it is obtained the version for the ordinary Riemann integral

$$
\left|\frac{X(a, \cdot)+X(b, \cdot)}{2}-\frac{1}{(b-a)} \int_{a}^{b} X(t, \cdot) d t\right| \leq \frac{(b-a)^{2}}{8} \sup _{\xi \in[a, b]}\left|X^{\prime \prime}(\xi, \cdot)\right|,
$$

almost everywhere.

Vol. 36, $\left.\mathrm{N}^{\circ} 2,2018\right]$ 
Theorem 3.5. Let $\alpha>0$ and $\rho>0$. Let $X:\left[a^{\rho}, b^{\rho}\right] \times \Omega \rightarrow \mathbb{R}$ be a mean square differentiable stochastic process with $0 \leq a<b$ and $X(t, \cdot) \in X_{c}^{p}\left(a^{\rho}, b^{\rho}\right)$. If $\left|X^{\prime}(t, \cdot)\right|$ is convex, then the following inequality holds almost everywhere:

$$
\begin{array}{r}
\left|\frac{X\left(a^{\rho}, \cdot\right)+X\left(b^{\rho}, \cdot\right)}{2}-\frac{\alpha \rho^{\alpha} \Gamma(\alpha+1)}{2\left(b^{\rho}-a^{\rho}\right)^{\alpha}}\left({ }^{\rho} I_{b^{\rho}-}^{\alpha} X\left(a^{\rho}, \cdot\right)+{ }^{\rho} I_{a^{\rho}+}^{\alpha} X\left(b^{\rho}, \cdot\right)\right)\right| \\
\leq \frac{\left(b^{\rho}-a^{\rho}\right)\left(\left|X^{\prime}\left(a^{\rho} \cdot\right)\right|+\left|X^{\prime}\left(b^{\rho} \cdot\right)\right|\right)}{2(\alpha+1)} .
\end{array}
$$

Proof. Using equality (11), the triangular inequality and the convexity of $\left|X^{\prime}(t, \cdot)\right|$, it is obtained that

$$
\begin{aligned}
& \left|\frac{X\left(a^{\rho}, \cdot\right)+X\left(b^{\rho}, \cdot\right)}{\alpha \rho}-\frac{\Gamma(\alpha+1)}{2 \rho^{1-\alpha}\left(b^{\rho}-a^{\rho}\right)^{\alpha}}\left({ }^{\rho} I_{b^{\rho}-}^{\alpha} X\left(a^{\rho}, \cdot\right)+{ }^{\rho} I_{a^{\rho}+}^{\alpha} X\left(b^{\rho}, \cdot\right)\right)\right| \\
& \leq \frac{\left(b^{\rho}-a^{\rho}\right)}{\alpha} \int_{0}^{1} t^{\rho(\alpha+1)-1} \mid X^{\prime}\left(t^{\rho} a^{\rho}+\left(1-t^{\rho}\right) b^{\alpha}, \cdot\right) \\
& \quad-X^{\prime}\left(\left(1-t^{\rho}\right) a^{\rho}+t^{\rho} b^{\rho}, \cdot\right) \mid d t \\
& \leq \frac{\left(b^{\rho}-a^{\rho}\right)}{\alpha}\left(\int_{0}^{1} t^{\rho(\alpha+1)-1}\left|X^{\prime}\left(t^{\rho} a^{\rho}+\left(1-t^{\rho}\right) b^{\alpha}, \cdot\right)\right| d t\right. \\
& \left.\quad+\int_{0}^{1} t^{\rho(\alpha+1)-1}\left|X^{\prime}\left(\left(1-t^{\rho}\right) a^{\rho}+t^{\rho} b^{\rho}, \cdot\right)\right| d t\right) \\
& \leq \frac{\left(b^{\rho}-a^{\rho}\right)}{\alpha}\left(\int_{0}^{1} t^{\rho(\alpha+1)-1}\left(t^{\rho}\left|X^{\prime}\left(a^{\rho} \cdot\right)\right|+\left(1-t^{\rho}\right)\left|X^{\prime}\left(b^{\rho} \cdot\right)\right|\right) d t\right. \\
& \left.\quad+\int_{0}^{1} t^{\rho(\alpha+1)-1}\left(\left(1-t^{\rho}\right)\left|X^{\prime}\left(a^{\rho} \cdot\right)\right|+t^{\rho}\left|X^{\prime}\left(b^{\rho} \cdot\right)\right|\right) d t\right) \\
& =\frac{\left(b^{\rho}-a^{\rho}\right)\left(\left|X^{\prime}\left(a^{\rho} \cdot\right)\right|+\left|X^{\prime}\left(b^{\rho} \cdot\right)\right|\right)}{\alpha} \int_{0}^{1} t^{\rho(\alpha+1)-1} d t \\
& =\frac{\left(b^{\rho}-a^{\rho}\right)\left(\left|X^{\prime}\left(a^{\rho} \cdot\right)\right|+\left|X^{\prime}\left(b^{\rho} \cdot\right)\right|\right)}{\alpha \rho(\alpha+1)} .
\end{aligned}
$$

The proof is complete.

Remark 3.6. With the same reasoning used in Remarks 3.2 and 3.4, it is obtained the following inequality almost everywhere, for the Riemann-Liouville fractional integral:

$$
\begin{aligned}
\mid \frac{X(a, \cdot)+X(b, \cdot)}{2}-\frac{\Gamma(\alpha+1)}{2(b-a)^{\alpha}}\left(J_{b-}^{\alpha} X(a, \cdot)\right. & \left.+J_{a+}^{\alpha} X(b, \cdot)\right) \mid \\
\leq & \frac{(b-a)\left(\left|X^{\prime}(a \cdot)\right|+\left|X^{\prime}(b \cdot)\right|\right)}{2(\alpha+1)} .
\end{aligned}
$$

Letting $\alpha=1$ in (13), it is obtained the inequality for the ordinary Riemann integral

$$
\left|\frac{X(a, \cdot)+X(b, \cdot)}{2}-\frac{1}{(b-a)} \int_{a}^{b} X(t, \cdot) d t\right| \leq \frac{(b-a)\left(\left|X^{\prime}(a \cdot)\right|+\left|X^{\prime}(b \cdot)\right|\right)}{4} .
$$

[Revista Integración, temas de matemáticas 
Lemma 3.7. Let $X:\left[a^{\rho}, b^{\rho}\right] \times \Omega \rightarrow \mathbb{R}$ be a mean square differentiable stochastic process; then the following equality holds:

$$
\begin{aligned}
\frac{X\left(a^{\rho}, \cdot\right)+X\left(b^{\rho}, \cdot\right)}{2}- & \frac{\alpha \rho^{\alpha} \Gamma(\alpha+1)}{2\left(b^{\rho}-a^{\rho}\right)^{\alpha}}\left({ }^{\rho} I_{b^{\rho}-}^{\alpha} X\left(a^{\rho}, \cdot\right)+{ }^{\rho} I_{a^{\rho}+}^{\alpha} X\left(b^{\rho}, \cdot\right)\right) \\
& =\frac{b^{\rho}-a^{\rho}}{2} \int_{0}^{1}\left[\left(1-t^{\rho}\right)^{\alpha}-t^{\rho \alpha}\right] t^{\rho-1} X^{\prime}\left(t^{\rho} a^{\rho}+\left(1-t^{\rho}\right) b^{\rho},\right) d t .
\end{aligned}
$$

Proof. First, it must be noted that

$$
\begin{aligned}
\int_{0}^{1}\left[\left(1-t^{\rho}\right)^{\alpha}-t^{\rho \alpha}\right] & t^{\rho-1} X^{\prime}\left(t^{\rho} a^{\rho}+\left(1-t^{\rho}\right) b^{\rho},\right) d t \\
= & \int_{0}^{1}\left(1-t^{\rho}\right)^{\alpha} t^{\rho-1} X^{\prime}\left(t^{\rho} a^{\rho}+\left(1-t^{\rho}\right) b^{\rho},\right) d t \\
& -\int_{0}^{1} t^{\rho(\alpha+1)-1} X^{\prime}\left(t^{\rho} a^{\rho}+\left(1-t^{\rho}\right) b^{\rho},\right) d t
\end{aligned}
$$

and using integration by parts it is obtained

$$
\begin{gathered}
\int_{0}^{1}\left(1-t^{\rho}\right)^{\alpha} t^{\rho-1} X^{\prime}\left(t^{\rho} a^{\rho}+\left(1-t^{\rho}\right) b^{\rho},\right) d t \\
=\left.\frac{\left(1-t^{\rho}\right)^{\alpha} X\left(t^{\rho} a^{\rho}+\left(1-t^{\rho}\right) b^{\rho},\right)}{\rho\left(a^{\rho}-b^{\rho}\right)}\right|_{0} ^{1} \\
\quad+\frac{\alpha}{a^{\rho}-b^{\rho}} \int_{0}^{1}\left(1-t^{\rho}\right)^{\alpha} t^{\rho-1} X\left(t^{\rho} a^{\rho}+\left(1-t^{\rho}\right) b^{\rho},\right) d t \\
=\frac{X\left(b^{\rho},\right)}{\rho\left(a^{\rho}-b^{\rho}\right)}-\frac{\alpha \rho^{\alpha} \Gamma(\alpha+1)^{\rho}}{\left(b^{\rho}-a^{\rho}\right)^{\alpha}} I_{b^{\rho}-}^{\alpha} X\left(a^{\rho}, \cdot\right),
\end{gathered}
$$

and similarly,

$$
\int_{0}^{1} t^{\rho(\alpha+1)-1} X^{\prime}\left(t^{\rho} a^{\rho}+\left(1-t^{\rho}\right) b^{\rho},\right) d t=-\frac{X\left(a^{\rho},\right)}{\rho\left(a^{\rho}-b^{\rho}\right)}+\frac{\alpha \rho^{\alpha} \Gamma(\alpha+1)^{\rho}}{\left(b^{\rho}-a^{\rho}\right)^{\alpha}} I_{b^{\rho}-}^{\alpha} X\left(b^{\rho}, \cdot\right) ;
$$

adding these last results we get the desired result.

The proof is complete.

Theorem 3.8. Let $X:\left[a^{\rho}, b^{\rho}\right] \times \Omega \rightarrow \mathbb{R}$ be a mean square differentiable stochastic process on $\left[a^{\rho}, b^{\rho}\right]$. If $\left|X^{\prime}\right|$ is a convex stochastic process, then the following inequality holds almost everywhere:

$$
\begin{aligned}
& \left|\frac{X\left(a^{\rho}, \cdot\right)+X\left(b^{\rho}, \cdot\right)}{2}-\frac{\alpha \rho^{\alpha} \Gamma(\alpha+1)}{2\left(b^{\rho}-a^{\rho}\right)^{\alpha}}\left({ }^{\rho} I_{b^{\rho}-}^{\alpha} X\left(a^{\rho}, \cdot\right)+{ }^{\rho} I_{a^{\rho}+}^{\alpha} X\left(b^{\rho}, \cdot\right)\right)\right| \\
& \leq \frac{b^{\rho}-a^{\rho}}{2 \rho(\alpha+1)}\left(1-\frac{\left(\frac{1}{2}\right)^{\alpha}}{\alpha+1}\right)\left(\left|X^{\prime}(a, \cdot)\right|+\left|X^{\prime}(b, \cdot)\right|\right) .
\end{aligned}
$$

Vol. 36, $\left.\mathrm{N}^{\circ} 2,2018\right]$ 
Proof. Using Lemma 3.7 and the convexity of $\left|X^{\prime}\right|$, it is obtained that

$$
\begin{aligned}
& \left|\frac{X\left(a^{\rho}, \cdot\right)+X\left(b^{\rho}, \cdot\right)}{2}-\frac{\alpha \rho^{\alpha} \Gamma(\alpha+1)}{2\left(b^{\rho}-a^{\rho}\right)^{\alpha}}\left({ }^{\rho} I_{b^{\rho}-}^{\alpha} X\left(a^{\rho}, \cdot\right)+{ }^{\rho} I_{a^{\rho}+}^{\alpha} X\left(b^{\rho}, \cdot\right)\right)\right| \\
\leq & \frac{b^{\rho}-a^{\rho}}{2} \int_{0}^{1}\left|\left(1-t^{\rho}\right)^{\alpha}-t^{\rho \alpha}\right| t^{\rho-1}\left|X^{\prime}\left(t^{\rho} a^{\rho}+\left(1-t^{\rho}\right) b^{\rho},\right)\right| d t \\
\leq & \frac{b^{\rho}-a^{\rho}}{2} \int_{0}^{1}\left|\left(1-t^{\rho}\right)^{\alpha}-t^{\rho \alpha}\right| t^{\rho-1}\left(t^{\rho}\left|X^{\prime}\left(a^{\rho}, \cdot\right)\right|+\left(1-t^{\rho}\right)\left|X^{\prime}\left(b^{\rho}, \cdot\right)\right|\right) d t \\
= & \frac{b^{\rho}-a^{\rho}}{2} \times \\
& \left(\int_{0}^{1 / 2^{1 / \rho}}\left(\left(1-t^{\rho}\right)^{\alpha}-t^{\rho \alpha}\right) t^{\rho-1}\left(t^{\rho}\left|X^{\prime}\left(a^{\rho}, \cdot\right)\right|+\left(1-t^{\rho}\right)\left|X^{\prime}\left(b^{\rho}, \cdot\right)\right|\right) d t\right. \\
& \left.+\int_{1 / 2^{1 / \rho}}^{1}\left(t^{\rho \alpha}-\left(1-t^{\rho}\right)^{\alpha}\right) t^{\rho-1}\left(t^{\rho}\left|X^{\prime}\left(a^{\rho}, \cdot\right)\right|+\left(1-t^{\rho}\right)\left|X^{\prime}\left(b^{\rho}, \cdot\right)\right|\right) d t\right) \\
& =\frac{b^{\rho}-a^{\rho}}{2} \times \\
& \left(\int_{0}^{1}\left(t^{\rho \alpha}-\left(1-t^{\rho}\right)^{\alpha}\right) t^{\rho-1}\left(t^{\rho}\left|X^{\prime}\left(a^{\rho}, \cdot\right)\right|+\left(1-t^{\rho}\right)\left|X^{\prime}\left(b^{\rho}, \cdot\right)\right|\right) d t\right. \\
- & \left.2 \int_{0}^{1 / 2^{1 / \rho}}\left(t^{\rho \alpha}-\left(1-t^{\rho}\right)^{\alpha}\right) t^{\rho-1}\left(t^{\rho}\left|X^{\prime}\left(a^{\rho}, \cdot\right)\right|+\left(1-t^{\rho}\right)\left|X^{\prime}\left(b^{\rho}, \cdot\right)\right|\right) d t\right) .
\end{aligned}
$$

Making the corresponding substitution in the previous inequalities, we have:

$$
\begin{aligned}
\int_{0}^{1}\left(t^{\rho \alpha}-\left(1-t^{\rho}\right)^{\alpha}\right) t^{\rho-1} & \left(t^{\rho}\left|X^{\prime}\left(a^{\rho}, \cdot\right)\right|+\left(1-t^{\rho}\right)\left|X^{\prime}\left(b^{\rho}, \cdot\right)\right|\right) d t \\
= & \left|X^{\prime}\left(a^{\rho}, \cdot\right)\right|\left(\int_{0}^{1} t^{\rho \alpha} t^{\rho-1} t^{\rho} d t-\int_{0}^{1}\left(1-t^{\rho}\right)^{\alpha} t^{\rho-1} t^{\rho} d t\right) \\
& -\left|X^{\prime}\left(b^{\rho}, \cdot\right)\right|\left(\int_{0}^{1} t^{\rho \alpha} t^{\rho-1}\left(1-t^{\rho}\right) d t-\int_{0}^{1} t^{\rho-1}\left(1-t^{\rho}\right)^{\alpha+1} d t\right) .
\end{aligned}
$$

With the change of variable $x=t^{\rho}$ it is obtained

$$
\begin{aligned}
\int_{0}^{1}\left(t^{\rho \alpha}-\left(1-t^{\rho}\right)^{\alpha}\right) t^{\rho-1}\left(t^{\rho}\left|X^{\prime}\left(a^{\rho}, \cdot\right)\right|+\left(1-t^{\rho}\right)\left|X^{\prime}\left(b^{\rho}, \cdot\right)\right|\right) d t & \\
= & \left(\left|X^{\prime}\left(a^{\rho}, \cdot\right)\right|-\left|X^{\prime}\left(b^{\rho}, \cdot\right)\right|\right)\left(\frac{\alpha}{\rho(\alpha+1)(\alpha+2)}\right),
\end{aligned}
$$

and similarly,

$$
\begin{aligned}
& \int_{0}^{1 / 2^{1 / \rho}}\left(t^{\rho \alpha}-\left(1-t^{\rho}\right)^{\alpha}\right) t^{\rho-1}\left(t^{\rho}\left|X^{\prime}\left(a^{\rho}, \cdot\right)\right|+\left(1-t^{\rho}\right)\left|X^{\prime}\left(b^{\rho}, \cdot\right)\right|\right) d t \\
& \quad=\left(\left|X^{\prime}\left(a^{\rho}, \cdot\right)\right|+\left|X^{\prime}\left(b^{\rho}, \cdot\right)\right|\right)\left(\frac{\left(\frac{1}{2}\right)^{\alpha+1}}{\rho(\alpha+1)}\right)-\frac{\left|X^{\prime}\left(a^{\rho}, \cdot\right)\right|}{(\alpha+1)(\alpha+2)}-\frac{\left|X^{\prime}\left(b^{\rho}, \cdot\right)\right|}{\alpha+2} .
\end{aligned}
$$

[Revista Integración, temas de matemáticas 
So,

$$
\begin{aligned}
& \left|\frac{X\left(a^{\rho}, \cdot\right)+X\left(b^{\rho}, \cdot\right)}{2}-\frac{\alpha \rho^{\alpha} \Gamma(\alpha+1)}{2\left(b^{\rho}-a^{\rho}\right)^{\alpha}}\left({ }^{\rho} I_{b^{\rho}-}^{\alpha} X\left(a^{\rho}, \cdot\right)+{ }^{\rho} I_{a^{\rho}+}^{\alpha} X\left(b^{\rho}, \cdot\right)\right)\right| \\
& \leq \frac{b^{\rho}-a^{\rho}}{2}\left\{\left(\left|X^{\prime}\left(a^{\rho}, \cdot\right)\right|-\left|X^{\prime}\left(b^{\rho}, \cdot\right)\right|\right)\left(\frac{\alpha}{\rho(\alpha+1)(\alpha+2)}\right)\right. \\
& \left.\quad-2\left[\left(\left|X^{\prime}\left(a^{\rho}, \cdot\right)\right|+\left|X^{\prime}\left(b^{\rho}, \cdot\right)\right|\right)\left(\frac{\left(\frac{1}{2}\right)^{\alpha+1}}{\rho(\alpha+1)}\right)-\frac{\left|X^{\prime}\left(a^{\rho}, \cdot\right)\right|}{(\alpha+1)(\alpha+2)}-\frac{\left|X^{\prime}\left(b^{\rho}, \cdot\right)\right|}{\alpha+2}\right]\right\} \\
& =\frac{b^{\rho}-a^{\rho}}{2 \rho(\alpha+1)}\left(1-\frac{\left(\frac{1}{2}\right)^{\alpha}}{\alpha+1}\right)\left(\left|X^{\prime}(a, \cdot)\right|+\left|X^{\prime}(b, \cdot)\right|\right) .
\end{aligned}
$$

The proof is complete.

Remark 3.9. With the same reasoning used in Remarks 3.2, 3.4 and 3.6, it is obtained the following inequality almost everywhere, for the Riemann-Liouville fractional integral:

$$
\begin{aligned}
\mid \frac{X(a, \cdot)+X(b, \cdot)}{2}-\frac{\Gamma(\alpha+1)}{2(b-a)^{\alpha}} & \left(J_{b-}^{\alpha} X(a, \cdot)+J_{a+}^{\alpha} X(b, \cdot)\right) \mid \\
& \leq \frac{b-a}{2}\left(1-\frac{\left(\frac{1}{2}\right)^{\alpha}}{\alpha+1}\right)\left(\left|X^{\prime}(a, \cdot)\right|+\left|X^{\prime}(b, \cdot)\right|\right) .
\end{aligned}
$$

Letting $\alpha=1$ in (14), it is obtained the following inequality, almost everywhere, for the ordinary Riemann integral:

$$
\left|\frac{X(a, \cdot)+X(b, \cdot)}{2}-\frac{1}{(b-a)} \int_{a}^{b} X(t, \cdot) d t\right| \leq \frac{3(b-a)}{8}\left(\left|X^{\prime}(a, \cdot)\right|+\left|X^{\prime}(b, \cdot)\right|\right) .
$$

Next theorem proposes a refinement of the Hermite-Hadamard inequality using Katugampola fractional integral.

Theorem 3.10. Let $\alpha>0$ and $\rho>0$. Let $X:\left[a^{\rho}, b^{\rho}\right] \times \Omega \rightarrow \mathbb{R}$ be a positive stochastic process with $0 \leq a<b$ and $X(t, \cdot) \in X_{c}^{p}\left(a^{\rho}, b^{\rho}\right)$. If $X(t, \cdot)$ is Jensen-convex and mean square continuous in the interval $\left[a^{\rho}, b^{\rho}\right]$, the following inequality holds almost everywhere:

$$
\begin{aligned}
& X\left(\frac{a^{\rho}+b^{\rho}}{2}, \cdot\right) \\
& \quad \leq h(\lambda) \leq \frac{\Gamma(\alpha+1)}{2 \rho^{-\alpha}\left(v^{\rho}-u^{\rho}\right)^{\alpha}}\left({ }^{\rho} \mathcal{S}_{1}^{\alpha}[u, v ; \lambda ; X]+{ }^{\rho} \mathcal{S}_{2}^{\alpha}[v, u ; \lambda ; X]\right) \\
& \quad \leq H(\lambda) \leq \frac{X\left(a^{\rho}, \cdot\right)+X\left(b^{\rho}, \cdot\right)}{2 \rho \alpha},
\end{aligned}
$$

where

$$
h(\lambda)=\lambda^{\rho} X\left(\frac{\left(2-\lambda^{\rho}\right) u^{\rho}+\lambda^{\rho} v^{\rho}}{2}, \cdot\right)+\left(1-\lambda^{\rho}\right) X\left(\frac{\left(1+\lambda^{\rho}\right) u^{\rho}+\left(1-\lambda^{\rho}\right) v^{\rho}}{2}, \cdot\right),
$$

Vol. 36, $\mathrm{N}^{\circ}$ 2, 2018] 


$$
\begin{aligned}
& H(\lambda)=\lambda^{\rho} \frac{X\left(u^{\rho}, \cdot\right)+X\left(\lambda^{\rho} v^{\rho}+\left(1-\lambda^{\rho}\right) u^{\rho}, \cdot\right)}{2 \rho \alpha} \\
& +\left(1-\lambda^{\rho}\right) \frac{X\left(\lambda^{\rho} v^{\rho}+\left(1-\lambda^{\rho}\right) u^{\rho}, \cdot\right)+X\left(v^{\rho}, \cdot\right)}{2 \rho \alpha}, \\
& { }^{\rho} \mathcal{S}_{1}^{\alpha}[u, v ; \lambda ; X]={ }^{\rho} I_{\lambda^{\rho} v^{\rho}+\left(1-\lambda^{\rho}\right) u^{\rho}-}^{\alpha} X\left(u^{\rho}, \cdot\right)+{ }^{\rho} I_{u^{\rho}+}^{\alpha} X\left(\lambda^{\rho} v^{\rho}+\left(1-\lambda^{\rho}\right) u^{\rho}, \cdot\right)
\end{aligned}
$$

and

$$
{ }^{\rho} \mathcal{S}_{1}^{\alpha}[v, u ; \lambda ; X]={ }^{\rho} I_{v^{\rho}-}^{\alpha} X\left(\lambda^{\rho} v^{\rho}+\left(1-\lambda^{\rho}\right) u^{\rho}, \cdot\right)+{ }^{\rho} I_{\lambda^{\rho} v^{\rho}+\left(1-\lambda^{\rho}\right) u^{\rho}+}^{\alpha} X\left(v^{\rho}, \cdot\right) .
$$

Proof. Applying (2) in the interval $\left[u^{\rho}, \lambda^{\rho} v^{\rho}+\left(1-\lambda^{\rho}\right) u^{\rho}\right]$, we have:

$$
\begin{gathered}
X\left(\frac{\left(2-\lambda^{\rho}\right) u^{\rho}+\lambda^{\rho} v^{\rho}}{2}, \cdot\right) \\
\leq \frac{\Gamma(\alpha+1)}{2 \rho^{-\alpha} \lambda^{\rho}\left(v^{\rho}-u^{\rho}\right)^{\alpha}} \times \\
\\
\left({ }^{\rho} I_{\lambda^{\rho}}^{\alpha} v^{\rho}+\left(1-\lambda^{\rho}\right) u^{\rho}-X\left(u^{\rho}, \cdot\right)+{ }^{\rho} I_{u^{\rho}+}^{\alpha} X\left(\lambda^{\rho} v^{\rho}+\left(1-\lambda^{\rho}\right) u^{\rho}, \cdot\right)\right) \\
\leq \\
\leq \frac{X\left(u^{\rho}, \cdot\right)+X\left(\lambda^{\rho} v^{\rho}+\left(1-\lambda^{\rho}\right) u^{\rho}, \cdot\right)}{2 \rho \alpha} .
\end{gathered}
$$

Now, for the interval $\left[\lambda^{\rho} v^{\rho}+\left(1-\lambda^{\rho}\right) u^{\rho}, v^{\rho}\right]$ we have:

$$
\begin{gathered}
X\left(\frac{\left(1+\lambda^{\rho}\right) u^{\rho}+\left(1-\lambda^{\rho}\right) v^{\rho}}{2}, \cdot\right) \\
\leq \frac{\Gamma(\alpha+1)}{2 \rho^{-\alpha}\left(1-\lambda^{\rho}\right)\left(v^{\rho}-u^{\rho}\right)^{\alpha}} \times \\
\\
\leq \frac{\left({ }^{\rho} I_{v^{\rho}-}^{\alpha} X\left(\lambda^{\rho} v^{\rho}+\left(1-\lambda^{\rho}\right) u^{\rho}, \cdot\right)+{ }^{\rho} I_{\lambda^{\rho} v^{\rho}+\left(1-\lambda^{\rho}\right) u^{\rho}+}^{\alpha} X\left(v^{\rho}, \cdot\right)\right)}{2 \rho \alpha} .
\end{gathered}
$$

Multiplying (15) by $\lambda^{\rho}$ and (16) by $\left(1-\lambda^{\rho}\right)$, and adding these inequalities, we have

$$
h(\lambda) \leq \frac{\Gamma(\alpha+1)\left({ }^{\rho} \mathcal{S}_{1}^{\alpha}[u, v ; \lambda ; X]+{ }^{\rho} \mathcal{S}_{2}^{\alpha}[v, u ; \lambda ; X]\right)}{2 \rho^{-\alpha}\left(v^{\rho}-u^{\rho}\right)^{\alpha}} \leq H(\lambda),
$$

where

$$
\begin{array}{r}
h(\lambda)=\lambda^{\rho} X\left(\frac{\left(2-\lambda^{\rho}\right) u^{\rho}+\lambda^{\rho} v^{\rho}}{2}, \cdot\right)+\left(1-\lambda^{\rho}\right) X\left(\frac{\left(1+\lambda^{\rho}\right) u^{\rho}+\left(1-\lambda^{\rho}\right) v^{\rho}}{2}, \cdot\right) \\
\begin{array}{r}
H(\lambda)=\lambda^{\rho} \frac{X\left(u^{\rho}, \cdot\right)+X\left(\lambda^{\rho} v^{\rho}+\left(1-\lambda^{\rho}\right) u^{\rho}, \cdot\right)}{2 \rho \alpha} \\
+\left(1-\lambda^{\rho}\right) \frac{X\left(\lambda^{\rho} v^{\rho}+\left(1-\lambda^{\rho}\right) u^{\rho}, \cdot\right)+X\left(v^{\rho}, \cdot\right)}{2 \rho \alpha}
\end{array}
\end{array}
$$




$$
{ }^{\rho} \mathcal{S}_{1}^{\alpha}[u, v ; \lambda ; X]={ }^{\rho} I_{\lambda^{\rho} v^{\rho}+\left(1-\lambda^{\rho}\right) u^{\rho}-}^{\alpha} X\left(u^{\rho}, \cdot\right)+{ }^{\rho} I_{u^{\rho}+}^{\alpha} X\left(\lambda^{\rho} v^{\rho}+\left(1-\lambda^{\rho}\right) u^{\rho}, \cdot\right)
$$

and

$$
{ }^{\rho} \mathcal{S}_{1}^{\alpha}[v, u ; \lambda ; X]={ }^{\rho} I_{v^{\rho}-}^{\alpha} X\left(\lambda^{\rho} v^{\rho}+\left(1-\lambda^{\rho}\right) u^{\rho}, \cdot\right)+{ }^{\rho} I_{\lambda^{\rho} v^{\rho}+\left(1-\lambda^{\rho}\right) u^{\rho}+}^{\alpha} X\left(v^{\rho}, \cdot\right)
$$

Using Theorem 2.5 it is seen that

$$
\begin{aligned}
X\left(\frac{a+b}{2}, \cdot\right) & =X\left(\lambda \frac{\lambda v+(2-\lambda) u}{2}+(1-\lambda) \frac{(1+\lambda) v+(1-\lambda) u}{2}, \cdot\right) \\
& \leq \lambda X\left(\frac{\lambda v+(2-\lambda) u}{2}, \cdot\right)+(1-\lambda) X\left(\frac{(1+\lambda) v+(1-\lambda) u}{2}, \cdot\right) \\
& \leq \frac{1}{2}(X(\lambda v+(1-\lambda) u, \cdot)+\lambda X(u, \cdot)+(1-\lambda) X(v, \cdot)) \\
& \leq \frac{X(u, \cdot)+X(v, \cdot)}{2} .
\end{aligned}
$$

From (17) and (18) it is attained the desired result. The proof is complete.

\section{Conclusions}

In the present article, the fractional integral of Katugampola was used to find the Hermite-Hadamard inequality for convex stochastic processes (Theorem 3.1), as well as some other results that estimate the difference between the value of the fractional integral and the right side of such inequality (Theorems 3.3, 3.5, 3.8), as well as a refinement of the aforementioned inequality (Theorem 3.10). From the results found, the same were deduced for the particular cases of Riemann-Liouville fractional integral and Riemann integral. The authors hope that this work will serve as a stimulus for future research in the area.

\section{Acknowledgements}

The authors thank the Council for Scientific, Humanistic and Technological Development (Consejo de Desarrollo Científico, Humanístico y Tecnológico, CDCHT) of the Centroccidental University Lisandro Alvarado (Universidad Centroccidental Lisandro Alvarado, UCLA) for the technical support provided in the preparation of this article belonging to the project RAC-2018-1.

They also thank Dr. Miguel Vivas (from Pontificia Universidad Católica del Ecuador), for his valuable collaboration, and the arbitrators appointed for the evaluation of this article.

\section{References}

[1] Agahi H. and Babakhani A., "On fractional stochastic inequalities related to HermiteHadamard and Jensen types for convex stochastic processes ", Aequationes Math. 90 (2016), No. 5, 1035-1043.

Vol. 36, $\left.\mathrm{N}^{\circ} 2,2018\right]$ 
[2] Alomari M. and Darus M., "On The Hadamard's Inequality for Log-Convex Functions on the Coordinates", J. Inequal. Appl. (2009), Article ID 283147, 13 pp.

[3] Alomari M., Darus M., Dragomir S.S. and Cerone P., "Ostrowski type inequalities for functions whose derivatives are s-convex in the second sense", Appl. Math. Lett. 23 (2010), No. 9, 1071-1076.

[4] Bain A. and Crisan D., Fundamentals of Stochastic Filtering, Springer-Verlag, New York, 2009.

[5] Chen H. and Katugampola U.N., "Hermite-Hadamard and Hermite-Hadamard-Fejér type inequalities for generalized fractional integrals", J. Math. Anal. Appl. 446 (2017), No. 2, $1274-1291$.

[6] Cortés J.C., Jódar L. and Villafuerte L., "Numerical solution of random differential equations: A mean square approach", Math. Comput. Modelling 45 (2007), No. 7-8, 757-765.

[7] Dahmani Z., "New inequalities in fractional integrals", Int. J. Nonlinear Sci. 9 (2010), No. 4, 493-497.

[8] Dahmani Z., Tabharit L. and Taf S., "New generalisations of Gruss inequality using Riemann-Liouville fractional integrals", Bull. Math. Anal. Appl. 2 (2010), No. 3, 93-99.

[9] Devolder P., Janssen J. and Manca R., Basic stochastic processes. Mathematics and Statistics Series, ISTE, London, John Wiley and Sons, Inc., 2015.

[10] Gorenflo R. and Mainardi F., "Fractional Calculus: Integral and Differential Equations of Fractional Order", in CISM Courses and Lect. 378, Springer, Vienna (1997), 223-276.

[11] Hadamard J., "Essai sur l'etude des fonctions donnees par leur developpment de Taylor", J. Math. Pures Appl. 8 (1892), 101-186.

[12] Katugampola U.N., "New approach to a generalized fractional integral", Appl. Math. Comput. 218 (2011), No. 3, 860-865

[13] Katugampola U.N., "A new approach to generalized fractional derivatives", Bull. Math. Anal. Appl. 6 (2014), No. 4, 1-15.

[14] Katugampola U.N., "Mellin transforms of generalized fractional integrals and derivatives", Appl. Math. Comput. 257 (2015), 566-580.

[15] Kilbas A.A., Srivastava H.M. and Trujillo J.J., Theory and Applications of Fractional Differential Equations, North-Holland Mathematical Studies, 204, Elsevier, Amsterdam (2006).

[16] Kotrys D., "Hermite-Hadamard inequality for convex stochastic processes", Aequationes Math. 83 (2012), No. 1-2, 143-151

[17] Kotrys D., "Remarks on strongly convex stochastic processes", Aequationes Math. 86 (2013), No. 1-2, 91-98.

[18] Kumar P., "Hermite-Hadamard inequalities and their applications in estimating moments", in Inequality Theory and Applications, Volume 2, (Edited by Y.J. Cho, J.K. Kim and S.S. Dragomir), Nova Science, (2003). 
[19] Liu W., Wen W. and J. Park, "Hermite-Hadamard type inequalities for MT-convex functions via classical integrals and fractional integrals", J. Nonlinear Sci. Appl. 9 (2016), No. $3,766-777$.

[20] Mikosch T., Elementary stochastic calculus with finance in view, Advanced Series on Statistical Science and Applied Probability, World Scientific Publishing Co., Inc., 2010.

[21] Miller S. and Ross B., An introduction to the Fractional Calculus and Fractional Diferential Equations, John Wiley \& Sons, USA, (1993).

[22] Mitrinovic D.S. and Lackovic I.B., "Hermite and convexity", Aequationes Math., 28 (1985), No. 1, 229-232.

[23] Nagy B., "On a generalization of the Cauchy equation", Aequationes Math. 10 (1974), No. $2-3,165-171$.

[24] Nikodem K., "On convex stochastic processes", Aequationes Math. 20 (1980), No. 1, 184197.

[25] Podlubny I., Fractional Differential Equations, Academic Press, San Diego, (1999).

[26] Sarikaya M.Z., Set E., Yaldiz H. and Basak N., "Hermite-Hadamard's inequalities for fractional integrals and related fractional inequalities", Math. Comput. Modelling 57 (2013), No. 9-10, 2403-2407.

[27] Set E., Tomar M. and Maden S., "Hermite Hadamard Type Inequalities for $s$-Convex Stochastic Processes in the Second Sense", Turkish Journal of Analysis and Number Theory 2 (2014), No. 6, 202-207.

[28] Set E., Akdemir A. and Uygun N., "On New Simpson Type Inequalities for Generalized Quasi-Convex Mappings", Xth International Statistics Days Conference, Giresun, Turkey, $571-581,2016$.

[29] Shaked M. and Shantikumar J. Stochastic Convexity and its Applications, Arizona Univ., Tucson, 1985.

[30] Shynk J.J., Probability, Random Variables, and Random Processes: Theory and Signal Processing Applications, John Wiley and Sons, Inc., 2013.

[31] Skowronski A., "On some properties of $J$-convex stochastic processes", Aequationes Math. 44 (1992), No. 2-3, 249-258.

[32] Skowronski A., "On Wright-Convex Stochastic Processes", Ann. Math. Sil. 9 (1995), 29-32.

[33] Thaiprayoon Ch., Ntouyas S., Tariboon J., "On the nonlocal Katugampola fractional integral conditions for fractional Langevin equation", Adv. Difference Equ. (2015) 2015:374, $16 \mathrm{pp}$.

[34] Tunç T., Budak H., Usta F. and Sarikaya M.Z, "On new generalized fractional integral operators and related inequalities", Submitted article, ResearchGate. https://www.researchgate.net/publication/313650587 [12 November 2018].

[35] Youness E.A., "E-convex sets, E-convex functions, and Econvex programming", J. Optim. Theory Appl. 102 (1999), No. 2, 439-450.

Vol. 36, $\left.\mathrm{N}^{\circ} 2,2018\right]$ 